\title{
Short and Mid-Term Economic Impact of Pulmonary Artery Catheter Use in Adult Cardiac Surgery: A Hospital and Integrated Health System Perspective
}

This article was published in the following Dove Press journal: ClinicoEconomics and Outcomes Research

\author{
Mitali Stevens' \\ Todd Davis' \\ Sibyl H Munson ${ }^{2}$ \\ Apeksha $V$ Shenoy ${ }^{2}$ \\ Boye LA Gricar ${ }^{2}$ \\ Halit O Yapici ${ }^{2}$ \\ Andrew D Shaw ${ }^{3}$ \\ 'Global Health Economics \& \\ Reimbursement, Edwards Lifesciences, \\ Irvine, CA, USA; ${ }^{2}$ Department of Health \\ Economics and Outcomes Research, \\ Boston Strategic Partners, Inc., Boston, \\ MA, USA; ${ }^{3}$ Department of \\ Anaesthesiology and Pain Medicine, \\ University of Alberta, Edmonton, \\ Alberta, Canada
}

Objective: A monitoring pulmonary artery catheter (PAC) is utilized in approximately $34 \%$ of the US cardiac surgical procedures. Increased use of PAC has been reported to have an association with complication rates: significant decreases in new-onset heart failure (HF) and respiratory failure $(\mathrm{RF})$, but increases in bacteremia and urinary tract infections. We assessed the impact of increasing PAC adoption on hospital costs among cardiac surgery patients for US-based healthcare systems.

Methods: An Excel-based economic model calculated annualized savings for a US hospital with various cardiac surgical volumes and PAC adoption rates. A second model, for an integrated payer-provider health system, analyzed outcomes/costs resulting from the cardiac surgical admission and for the treatment of persistent HF and RF complications in the year following surgery. Model inputs were extracted from published literature, and one-way and probabilistic sensitivity analyses were performed.

Results: For an acute care hospital with 500 procedures/year and 34\% PAC adoption, annualized savings equalled $\$ 61,806$ vs no PAC utilization. An increase in PAC adoption rate led to increased savings of $\$ 134,751$ for $75 \%$ and $\$ 170,685$ for $95 \%$ adoption. Savings ranged from $\$ 12,361$ to $\$ 185,418$ at volumes of 100 and 1500 procedures/year, respectively. For an integrated payer-provider health system with the base-case scenario of 3845 procedures/year and 34\% PAC adoption, estimated savings were \$596,637 for the combined surgical index admission and treatment for related complications over the following year.

Conclusion: PAC utilization in adult cardiac surgery patients results in reduced costs for both acute care hospitals and payer-provider integrated health systems.

Keywords: heart failure, respiratory failure, economic evaluation, cost analysis, acute care hospital, integrated payer-provider

\section{Introduction}

Cardiovascular surgery in the United States presents a sizable burden to the healthcare system with an annual number of coronary artery bypass graft (CABG) and valve procedures exceeding 290,000. ${ }^{1}$ The pulmonary artery catheter (PAC) may be used for simultaneous monitoring of multiple right heart pressures as well as continuous monitoring of cardiac output and mixed venous oxygen saturation, which makes it a useful tool for cardiac anesthesiologists. ${ }^{2}$ Although PAC use decreased between 1993 and $2004,{ }^{3}$ it is increasingly being used for heart surgeries, ${ }^{2,4}$ with a large study showing an increase in utilization from $26 \%$ in 2010 to $38 \%$ in 2014 among patients receiving $\mathrm{CABG}$ or valve procedures. ${ }^{2}$ Additionally, even with less invasive
Correspondence: Andrew D Shaw Department of Anaesthesiology and Pain Medicine, University of Alberta, 8440 II 2 St NW, Edmonton, Alberta T6G 2G3,

Canada

Email ashaw2@ualberta.ca 
hemodynamic monitoring devices and technologies available currently, a survey study by Judge et $\mathrm{al}^{4}$ found that most respondents preferred to place a PAC for monitoring during cardiac surgery.

Few studies have directly compared the costs for patients receiving a PAC versus not receiving a PAC within a US cardiac surgical population. Ramsey et $\mathrm{al}^{5}$ examined hospital costs for patients undergoing CABG procedures with and without PAC utilization, but the analysis was restricted to non-emergent cases, while Chiang et $\mathrm{al}^{6}$ examined only total hospital charges surrounding PAC use for CABG and valve procedures, which may not accurately reflect the direct cost to the hospital. Moreover, none of these studies evaluated the long-term costs associated with PAC utilization nor the longer-term economic impact of cardiopulmonary complications that may occur during the index surgical admission. Other studies evaluating economic outcomes for CABG and valve procedures included studies restricted to a particular PAC, ${ }^{7}$ partial costs, ${ }^{8}$ or were of non-US geography. ${ }^{9}$

A recent large matched cohort study used a US electronic health records (EHR) database to evaluate clinical outcomes associated with PAC use among patients undergoing all major cardiac procedures. ${ }^{10}$ PAC was associated with a significant decrease in the incidence of new-onset heart failure and respiratory failure following cardiac surgery, while the incidence of bacteremia and urinary tract infection significantly increased compared to the no-PAC group. ${ }^{10}$ The treatment of these conditions represents a significant economic burden in the short term. ${ }^{11-14}$ However, the economic impact for the treatment of heart failure and respiratory failure goes beyond the short term, ${ }^{15-18}$ unlike infections that can typically be treated in 5-14 days with antibiotics. ${ }^{19,20}$ The present study evaluated the economic impact of utilizing a monitoring PAC for adult cardiac surgeries from two perspectives: 1) the acute care hospital (index surgical admission); and 2) an integrated payer/provider health system which covers the cost of longer-term care (index surgical admission and the following year), to support decision-making processes regarding PAC adoption for patients undergoing cardiac surgical procedures.

\section{Methods}

\section{Model Overview}

Two economic models were built using Microsoft Excel (version 1906) to assess the impact of using a PAC for monitoring purposes in US cardiac surgeries. The first model looked at an acute care hospital focused on the cardiac surgical admission. The second model accounted for an integrated payer-provider health system (for payer-owned and operated hospitals) which included the surgical visit and mid-term costs for up to oneyear post-surgery. Both models calculated the annualized cost/ savings related to PAC use, as per their perspective, for a US hospital at various PAC adoption rates.

\section{Patient Population and Interventions}

Adult patients undergoing cardiac surgery who did or did not receive a PAC for monitoring purposes were included in the models. A large recent retrospective EHR study evaluating PAC use or non-use for adult cardiac procedures and the effects on clinical outcomes ${ }^{10}$ provided a robust propensity-matched study population for this analysis with 6844 patients analyzed. This study (NCT02964026) extracted HIPAA-compliant data from the US Cerner Health Facts ${ }^{\circledR}$ (Cerner Corp., Kansas City, MO, USA) database. In addition to hospital characteristics (bed size, teaching status, location) and encounterlevel patient data (demographics, admission type, payer), comprehensive timestamped medication orders, pharmacy records, laboratory results, admission and discharge diagnoses (International Classification of Diseases Ninth Revision Clinical Modification [ICD-9$\mathrm{CM}$ codes]), and procedures were available. ${ }^{10}$

The study population consisted of patients $\geq 18$ yearsof-age undergoing isolated CABG (64.8\%), isolated valve (20.5\%), and multi-cardiac procedures $(13.3 \%)$; $<2 \%$ consisted of other procedures (heart transplant, aortic, or other complex non-valvular surgeries) between January 1, 2011, and June 30, 2015. ${ }^{10}$ Patients were excluded if they received a percutaneous coronary intervention (PCI) prior to a $\mathrm{CABG}$ or valve procedure. See Shaw et $\mathrm{al}^{10}$ for detailed inclusion and exclusion criteria. Cohorts were assigned based on PAC usage, and patients without a monitoring PAC comprised the control arm. Patients fulfilling all inclusion/exclusion criteria were matched on patient and hospital demographics, surgery type, a modified European System for Cardiac Operative Risk Evaluation (EuroSCORE II), and Elixhauser comorbidities by a $1: 1$ propensity score method. ${ }^{10}$

Outcomes that were significantly different between the PAC and no-PAC cohorts were included in the model: new-onset heart failure, respiratory failure, bacteremia, and urine infection. The cardiopulmonary morbidity composite, one of the Shaw et al study primary outcomes, ${ }^{10}$ 
showed significantly improved outcomes for the PAC cohort primarily due to new-onset heart failure (HF), respiratory failure (RF), and haemorrhage (however, there was not a significant difference in transfusion rates, and thus haemorrhage was not included in the model). Conversely, the primary outcome of infectious morbidity composite favoured patients that did not receive a PAC, driven by a significant decrease in bacteremia and urine infection.

\section{Model and Analytic Framework Acute Care Hospital Model}

The impact on hospital costs with increasing PAC adoption among cardiac surgery patients was computed via annualized cost for institutions with varying cardiac surgical volumes. Based on the PAC adoption rate, the patient population for a given surgical volume was divided into PAC and "no-PAC" cohorts. The numbers of patients experiencing adverse clinical outcomes (new-onset heart failure, respiratory failure, bacteremia, and urinary tract infection) were calculated using cohort-specific complication rates from Shaw et al ${ }^{10}$ Complication-associated costs were estimated by multiplying the number of patients with each complication by their respective costs. Annual total PAC cost was determined by multiplying PAC device cost by the total annual number of procedures modelled with a PAC. For the acute care hospital model, annual costs/ savings were calculated by subtracting the costs for treatment of complications and total PAC device costs for a given ( $>0 \%)$ PAC adoption rate from costs associated with $0 \%$ PAC adoption.

\section{Integrated Payer-Provider Health System Model}

The integrated payer-provider health system model analyzed outcomes/costs from the cardiac surgical visit, as described above, as well as for the year following surgery. Mortality rates were applied to determine post-surgery survivors. Complications of HF and RF have long-term consequences for a proportion of patients, resulting in healthcare utilization and costs. ${ }^{21}$ Infectious complications of bacteremia and urine infection are treated during the hospital visit and do not typically require long-term treatment. ${ }^{19,20}$ The model calculated annual savings for a specific PAC adoption rate ( $>0 \%)$, by subtracting the costs for treatment of complications, total PAC device costs, and total patient visit costs in the year post-surgery for $>0 \%$ PAC utilization from the costs incurred with $0 \%$ PAC adoption.

\section{Model Inputs and Data Sources Acute Care Hospital Model}

Model inputs were obtained from multiple sources and are detailed in Table 1. Inputs for the acute care hospital model included an adult cardiac surgical load, PAC utilization rate, and cost for a monitoring PAC. PAC cost was calculated by taking the average cost of relevant PAC devices (pediatric PACs and pacing catheters/ probes were excluded) obtained from a market spend report. ${ }^{22}$ The rates of complications were based on the Shaw et $\mathrm{al}^{10}$ study. Inpatient-specific costs for each complication were determined from the US HCUP database (Agency for Healthcare Research and Quality, Rockville, MD). ${ }^{23}$ The ICD9 codes used and the query parameters for the HCUP database are found in Appendix Table 1. All costs were inflated to December 2018 US dollars (USD) using the United States Bureau of Labor and Statistics Consumer Price Index (CPI) inflation calculator. ${ }^{24}$

\section{Integrated Payer-Provider Health System Model}

The adult cardiac surgical load for the integrated payer-provider model was calculated for a large-size integrated health system in the US using cardiac surgical volume data from Kaiser Permanente-owned hospitals (3 states) serving a covered population of approximately 9.9 million (adult CABG and valve surgeries performed within Kaiser Permanente operated hospitals in the US states of CA, WA and HI). ${ }^{25,26}$ All remaining inputs for the index visit, including PAC utilization rate, PAC cost, index visit-related complication rates, and associated costs, were the same as within the acute care hospital model. Mortality rates for the surgical visit were extracted from Shaw et $\mathrm{al}^{10}$ study data with additional analysis (see Appendix Table 2). Rates for cardiac surgical patients with post-operative complications that persist (HF and RF) were extracted from published literature along with their respective healthcare utilization and costs. ${ }^{16,21,27,28}$

\section{Analyses and Outputs \\ Acute Care Hospital Model}

The base case assumed a medium cardiac surgical load $\left(\mathrm{n}=500\right.$ cases/year), a PAC utilization rate of $34.4 \%,{ }^{2}$ a mean PAC cost of $\$ 109.84,{ }^{22}$ and rates of complications as reported in Shaw et $\mathrm{al}^{10}$ at HCUP costs (Table 1). Acute care hospital model scenarios were executed for the following 
Table I Summary of Model Inputs

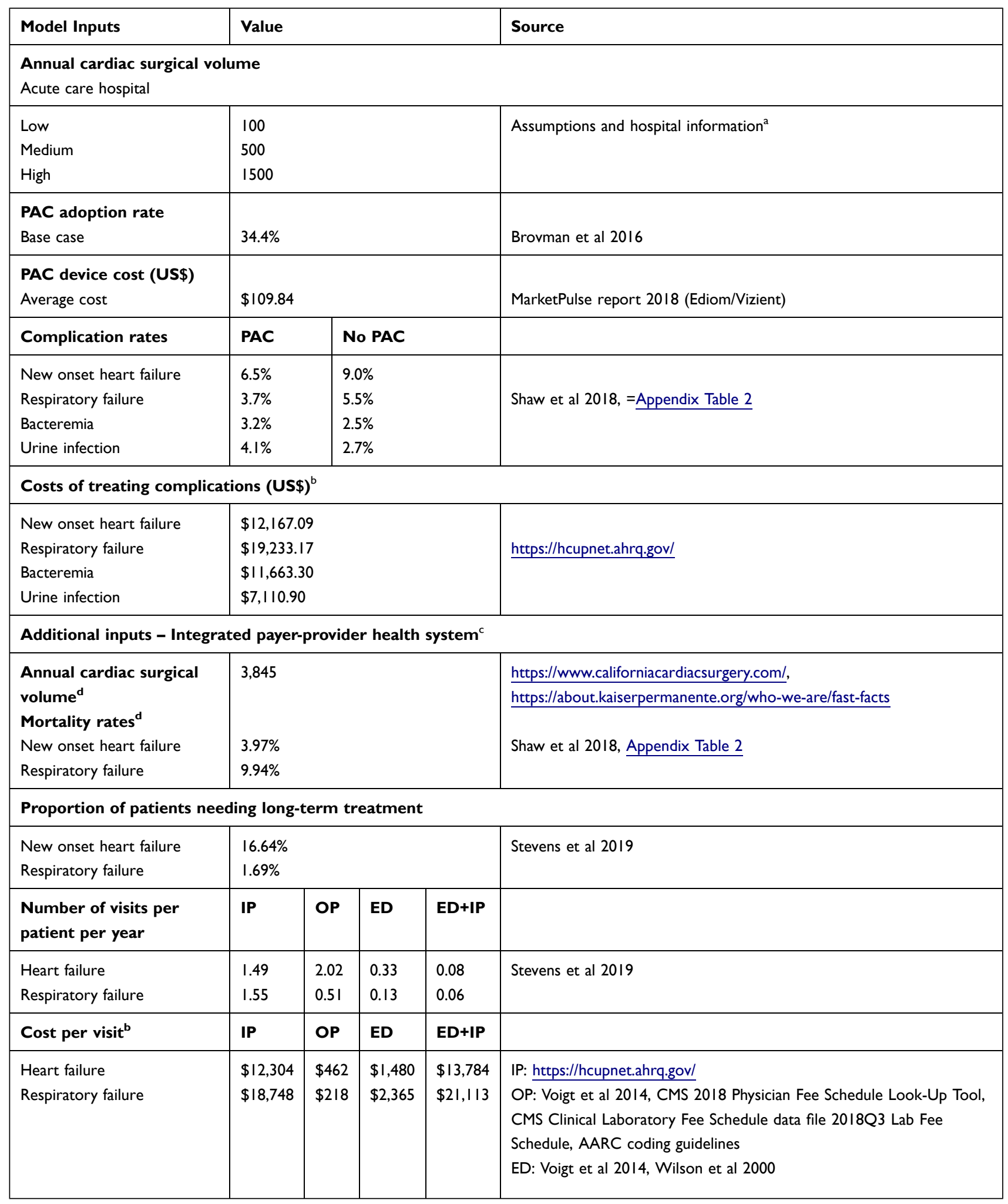

Notes: ${ }^{a}$ https://my.clevelandclinic.org/departments/heart/outcomes/359-surgical-treatment-for-ischemic-heart-disease https://medicine.umich.edu/sites/default/files/ CardiacOutcomes_2016.pdf. ${ }^{b}$ Values converted to 2018 using https://www.bls.gov/data/inflation_calculator.htm. ${ }^{c} A l l$ inputs for acute care are applicable except for surgical volume. ${ }^{\mathrm{d} S e e}$ Methods for details.

Abbreviations: IP, inpatient visit; OP, outpatient visit; ED, emergency department visit only; ED+IP, emergency department visit resulting in an inpatient stay. 
variable values: adult cardiac surgical load (low $=100 /$ year, medium $=500 /$ year, high $=1500 /$ year $)$ PAC utilization rate $(0.0 \%, 34.4 \%, 75.0 \%, 90.0 \%)$, and at a mean PAC cost of $\$ 109.84$. Clinical outcomes are reported for the base case, and economic outcomes are reported for varying cardiac surgical loads and PAC adoption rates.

\section{Integrated Payer-Provider Health System Model}

The base case was defined as 3845 cardiac surgeries, a PAC utilization rate of $34.4 \%,{ }^{2}$ a mean PAC cost of $\$ 109.84,{ }^{22}$ and rates of complications from Shaw et $\mathrm{al}^{10}$ with treatment costs derived from HCUP (Table 1). Mid-term costs up to one-year post-surgery were calculated across multiple visit types based on the persistence of complications post-surgery. Economic outcomes were inclusive of costs during the index surgical visit and over the one-year follow-up period for the proportion of patients that have persistent complications.

Annual savings for both models were calculated via a comparison of costs for a specified PAC utilization rate $(>0 \%)$ compared to costs resulting from no PAC adoption $(0 \%)$.

\section{Sensitivity Analyses One-Way Sensitivity Analysis}

A one-way sensitivity analysis was performed for the acute care hospital model. It evaluated the independent effects of each input (see Appendix Table 3) on the model outcome (total hospital costs/savings) for the index hospital admission. Inputs were varied independently using high and low input values. Lower and upper bounds were informed by published literature, where available, or varied $\pm 20 \%$ (complication rates) or $\pm 75 \%$ (cost data) (see Appendix Table 3).

\section{Probabilistic Sensitivity Analysis (PSA)}

A PSA was performed (@Risk add-in, version 7.6, Palisade, Ithaca, NY, USA) for both models to address the uncertainty of model inputs and provide a measure of confidence in the base case. All model inputs were varied simultaneously, for a total of 10,000 iterations. Input and assumption values were drawn from specified distributions (Appendix Table 4 [acute care hospital]; Appendix Table 5 [integrated payer-provider]). Distributions were parameterized using the mean and standard deviation. In cases where standard deviation was unknown, these were estimated using published methods. ${ }^{29}$

\section{Results}

\section{Acute Care Hospital Perspective}

Clinical and economic outcomes for an acute care hospital related to monitoring PAC usage among adult cardiac surgical patients are shown in Table 2 . While heart and respiratory failure complication rates are reduced ( $\mathrm{HF}$ : $9 \%$ to $6.6 \%, \mathrm{RF}$ : $5.5 \%$ to $3.8 \%$ ) with higher PAC usage ( $0 \%$ to $95 \%$, respectively), infection rates are increased (bacteremia: $2.5 \%$ to $3.2 \%$, urine infection: $2.7 \%$ to $4 \%$; $0 \%$ to $95 \%$ PAC adoption, respectively). Adoption of PAC for monitoring purposes led to annual savings that increased with hospital cardiac surgical load and PAC adoption rate (Figure 1). Savings resulted from decreased rates for $\mathrm{HF}$ and $\mathrm{RF}$, thus offsetting the PAC device cost, and costs for treating infections which are more frequent among patients that receive a PAC. For the base-case scenario, a hospital with an average cardiac surgical load of 500 cases and 34\% PAC adoption was estimated to have total annual savings of US $\$ 61,806.25$.

\section{One-Way Sensitivity Analysis}

A sensitivity analysis was performed to look at variation surrounding the base case (see Appendix Table 3 for inputs). Costs for treating $\mathrm{HF}$ and RF were the parameters with the greatest impact, followed by the complication rates of $\mathrm{HF}$ in the no PAC group, and for RF in the PAC group (see Appendix Figure 1). Savings were always positive and ranged from $\$ 8459$ for the lower bound PAC average sales price to $\$ 106,466$ for the higher bound RF treatment cost. Varying annual surgical loads had a relatively minor impact on total savings. Urine infection complication rates in the PAC and no PAC group had the least effect on savings.

\section{Probabilistic Sensitivity Analysis}

The overall mean total savings calculated from 10,000 iterations varying all input values simultaneously (see Appendix Table 4 for inputs) was $\$ 60,125.72$ (95\% CI \pm $\$ 973.48$; Figure 2A), close to the base-case savings $(\$ 61,806.25)$. Of the 10,000 model iterations, $91.9 \%$ of iterations generated savings between $\$ 1$ and $\$ 390,064.36$ (maximum). The PSA (Figure 2A), similar to the one-way sensitivity analysis, revealed that treatment costs for $\mathrm{HF}$ and RF had the largest impact on savings, followed by the PAC average sales price.

\section{Integrated Payer-Provider Health System Perspective}

Clinical and economic outcomes associated with increased PAC utilization for an integrated payer-provider health 
Table 2 Clinical and Economic Outcomes Associated with Increased PAC Usage in an Acute Care Hospital, Base Case

\begin{tabular}{|c|c|c|c|c|}
\hline & \multicolumn{4}{|c|}{ PAC Adoption Rates } \\
\hline & $0.0 \%$ & $34.4 \%$ & $75.0 \%$ & $95.0 \%$ \\
\hline \multicolumn{5}{|l|}{ Complications, $\%$ of cardiac surgical patients (n/year) ${ }^{\mathbf{b}}$} \\
\hline New onset heart failure & $9.0 \%(45)$ & $8.1 \%(4 I)$ & $7.1 \%(36)$ & $6.6 \%(33)$ \\
\hline Respiratory failure & $5.5 \%(28)$ & $4.9 \%(24)$ & $4.2 \%(21)$ & $3.8 \%(19)$ \\
\hline Bacteremia & $2.5 \%(13)$ & $2.7 \%(14)$ & $3.0 \%(15)$ & $3.2 \%(16)$ \\
\hline Urine infection & $2.7 \%(14)$ & $3.2 \%(16)$ & $3.8 \%(19)$ & $4.0 \%(20)$ \\
\hline \multicolumn{5}{|l|}{ Economic outcomes (US\$) } \\
\hline \multicolumn{5}{|l|}{ Cost of complications } \\
\hline New onset heart failure & $\$ 547,519.05$ & $\$ 495,200.56$ & $\$ 433,452.58$ & $\$ 403,034.86$ \\
\hline Respiratory failure & $\$ 528,912.18$ & $\$ 469,366.28$ & $\$ 399,088.28$ & $\$ 364,468.57$ \\
\hline Bacteremia & $\$ 145,791.25$ & $\$ 159,833.86$ & $\$ 176,407.41$ & $\$ 184,571.72$ \\
\hline Urine infection & $\$ 95,997.15$ & $\$ 113,120.20$ & $\$ 133,329.38$ & $\$ 143,284.64$ \\
\hline Total & $\$ 1,318,219.63$ & $\$ 1,237,520.90$ & $\$ 1,142,277.65$ & $\$ 1,095,359.79$ \\
\hline Total PAC cost (US\$) & $\$ 0$ & $\$ 18,892.48$ & $\$ 4 I, 190.00$ & $\$ 52,174.00$ \\
\hline Total cost for annual cardiac surgical volume (US\$) & $\$ 1,318,219.63$ & $\$ 1,256,4 \mid 3.38$ & $\$ 1,183,467.65$ & $\$ 1,147,533.79$ \\
\hline Total savings for annual cardiac surgical volume (US\$) ${ }^{c}$ & $\$ 0$ & $\$ 61,806.25$ & $\$ 134,751.98$ & $\$ 170,685.84$ \\
\hline
\end{tabular}

Notes: ${ }^{a}$ Annual cardiac surgical volume $=500$. ${ }^{b} \mathrm{n} /$ year rounded to nearest unit. ${ }^{c}$ Savings to the hospital compared to $0 \%$ PAC adoption.

system are shown in Table 3. Savings were largely due to lower HF and RF complication rates during the index visit as PAC adoption increased. A decrease in patients with $\mathrm{HF}$ and RF drove a reduction in healthcare utilization in the year following surgery, leading to additional health system savings. Overall savings increased as the PAC adoption rate increased. The base-case scenario, an average annual cardiac surgical load of 3845 procedures and a $34 \%$ PAC adoption rate, generated total annual savings of $\$ 596,637.18$, with index admission savings of $\$ 475,290.00$, and additional savings of $\$ 121,347.19$ in the year following surgery, compared to $0 \%$ PAC adoption.

\section{Probabilistic Sensitivity Analysis}

The overall mean total savings, calculated from 10,000 iterations varying input values simultaneously (see Appendix Table 5), were $\$ 584,343.69 \quad(95 \%$ CI \pm $\$ 8,206.90$ ), close to the base-case savings calculated as $\$ 596,637.18$. Ninety percent of model iterations generated positive savings between $\$ 20,334$ and $\$ 1,332,294$ (95.7\% yielded positive savings). PSA results (Figure 2B) demonstrated that treatment costs for HF and RF during the index visit had the largest impact on annualized savings, followed by complication rates for RF and HF for the no PAC group.

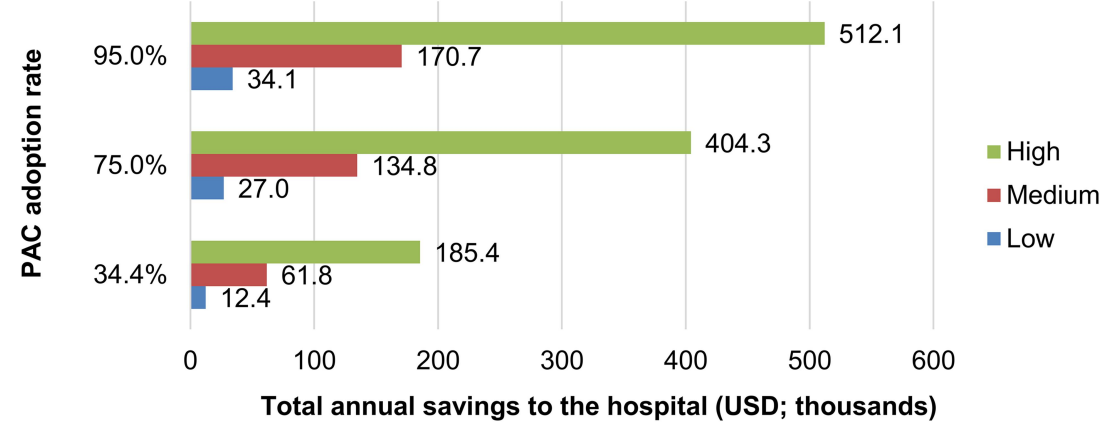

Figure I Total annual savings for an acute care hospital with varying annual cardiac surgery volume and PAC adoption rate, compared to $0 \%$ PAC adoption. Annual cardiac surgery load: low volume, 100; medium volume, 500; high volume, I,500.

Abbreviations: PAC, pulmonary artery catheter; USD, United States dollars. 


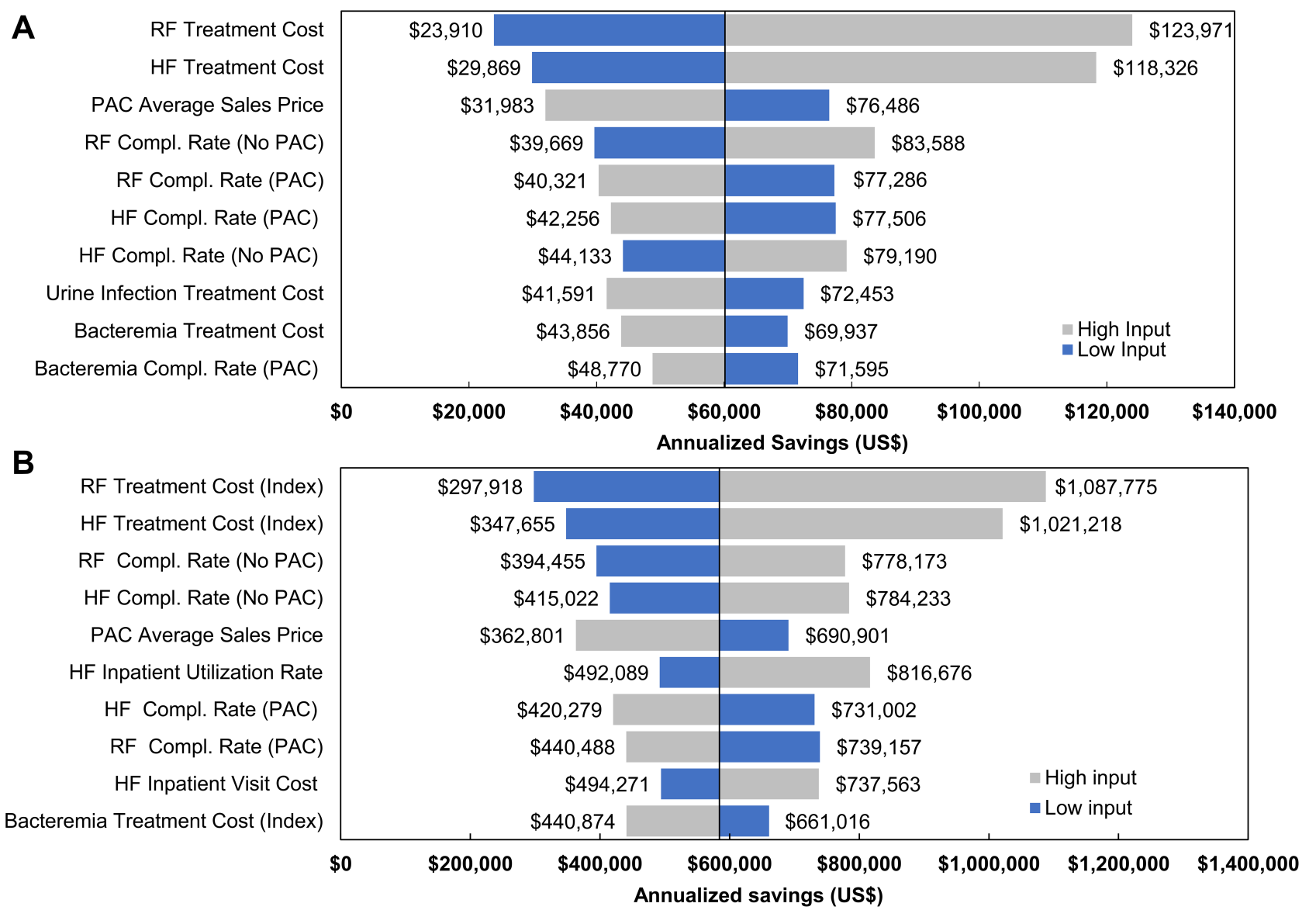

Figure 2 Probabilistic sensitivity analysis of total annual savings associated with $0.0 \%$ PAC adoption versus $34.4 \%$ PAC adoption ((A) acute care hospital, (B) integrated payer-provider). Inputs were sampled simultaneously from a specified distribution and ranked by their effect on the output mean. The length of each bar represents the change in total savings from the mean (a: $\$ 60,125.72,95 \% \mathrm{Cl} \pm \$ 973.48 ;$ b: $\$ 584,343.69 ; 95 \% \mathrm{Cl} \pm \$ 8,206.90$ ), represented by the midline. Grey bars represent savings associated with input of the upper bound, and blue bars represent savings associated with input of the lower bound for each variable. The 10 highest ranking inputs are shown. See Appendix Tables 4 and 5 for distribution parameters.

Abbreviations: Compl. rate, complication rate; HF, heart failure; PAC, pulmonary artery catheter; RF, respiratory failure.

\section{Discussion}

The economic models described in this study demonstrate that significant cost savings may be achieved by increasing the use of a monitoring PAC, presumably coupled with appropriate treatment, surrounding cardiac surgical procedures. Cost savings were in the range of $\$ 123.61$ to $\$ 341.37$ per cardiac surgical case for an acute care hospital and $\$ 155.17$ to $\$ 428.53$ per case for integrated payer-providers at $34 \%$ and $95 \%$ PAC adoption rates, respectively, inclusive of PAC device costs. From the acute care hospital perspective, monitoring PAC adoption resulted in annual savings which increased with higher rates of PAC adoption and greater annual numbers of surgical procedures. Savings are generated largely by a decrease in the frequency of $\mathrm{HF}$ and $\mathrm{RF}$ complications which offset both the PAC cost and the cost of treating infections, which occur more frequently in patients that receive a PAC. As expected, treatment costs for RF and HF complications that occur during the index surgical stay are higher than the cost of treating infections. Both deterministic and probabilistic sensitivity analyses demonstrated that RF treatment costs had the largest impact on savings, followed by HF treatment costs.

From the perspective of an integrated payer-provider, who is responsible for both the costs of the index admission and follow-up treatment, our study revealed annual savings of approximately $\$ 600,000$ for a $34 \%$ PAC adoption rate, and \$1.6 million for a 95\% PAC adoption rate. Similar to the acute hospital setting, savings were largely driven by a reduction in $\mathrm{HF}$ and $\mathrm{RF}$ complications during the index visit, with increased PAC adoption resulting in lower overall treatment cost. Probabilistic sensitivity analyses confirmed that the two inputs with the greatest 
Table 3 Clinical and Economic Outcomes Associated with Increased PAC Usage in an Integrated Payer-Provider System, Base Case ${ }^{a}$

\begin{tabular}{|c|c|c|c|c|}
\hline & \multicolumn{4}{|c|}{ PAC Adoption Rates } \\
\hline & $0.0 \%$ & $34.4 \%$ & $75.0 \%$ & $95.0 \%$ \\
\hline \multicolumn{5}{|l|}{ Index-visit summary } \\
\hline \multicolumn{5}{|c|}{ Complications, $\%$ of cardiac surgical patients (n/year) ${ }^{\mathbf{b}}$} \\
\hline New onset heart failure & $9.0 \%(346)$ & $8.1 \%(3 \mid 3)$ & $7.1 \%(274)$ & $6.6 \%(255)$ \\
\hline Respiratory failure & $5.5 \%(211)$ & $4.9 \%(188)$ & $4.2 \%(160)$ & $3.8 \%(146)$ \\
\hline Bacteremia & $2.5 \%(96)$ & $2.7 \%(105)$ & $3.0 \%(116)$ & $3.2 \%(122)$ \\
\hline Urine infection & $2.7 \%(104)$ & $3.2 \%(122)$ & $3.8 \%(144)$ & $4.0 \%(155)$ \\
\hline \multicolumn{5}{|l|}{ Economic outcomes (US\$) } \\
\hline \multicolumn{5}{|l|}{ Cost of complications } \\
\hline New onset heart failure & $\$ 4,210,421.49$ & $\$ 3,808,092.33$ & $\$ 3,333,250.35$ & $\$ 3,099,338.04$ \\
\hline Respiratory failure & $\$ 4,067,334.63$ & $\$ 3,609,426.70$ & $\$ 3,068,988.85$ & $\$ 2,802,763.31$ \\
\hline Bacteremia & $\$ 1,|21| 34.7 \mid$, & $\$ 1,229,|22.4|$ & $\$ 1,356,573.00$ & $\$ 1,419,356.55$ \\
\hline Urine infection & $\$ 738,218.08$ & $\$ 869,894.32$ & $\$ 1,025,302.89$ & $\$ 1,101,858.84$ \\
\hline Total & $\$ 10,137,108.92$ & $\$ 9,516,535.75$ & $\$ 8,784,115.10$ & $\$ 8,423,316.75$ \\
\hline Total PAC cost (US\$) & $\$ 0$ & $\$ 145,283.17$ & $\$ 316,751.10$ & $\$ 401,218.06$ \\
\hline Total cost for annual cardiac surgical volume (US\$) & $\$ 10,137,108.92$ & $\$ 9,661,818.92$ & $\$ 9,100,866.20$ & $\$ 8,824,534.8$ । \\
\hline \multicolumn{5}{|l|}{ Follow-up period (365-day) summary } \\
\hline \multicolumn{5}{|c|}{$\begin{array}{l}\text { Survivors, \% of patients surviving index hospitalization with } \\
\text { complications ( } n / \text { year) }\end{array}$} \\
\hline New onset heart failure & $96.0 \%(332)$ & $96.0 \%(301)$ & $96.0 \%(263)$ & $96.0 \%(245)$ \\
\hline Respiratory failure & $90.1 \%(190)$ & $90.1 \%(169)$ & $90.1 \%(144)$ & $90.1 \%(131)$ \\
\hline \multicolumn{5}{|l|}{ Persistent complications, $\%$ of survivors (n/year) ${ }^{b}$} \\
\hline Heart failure & $16.6 \%(55)$ & $16.6 \%(50)$ & $16.6 \%(44)$ & $16.6 \%(4 I)$ \\
\hline Respiratory failure & $1.7 \%(3)$ & $1.7 \%(3)$ & $1.7 \%(2)$ & $1.7 \%(2)$ \\
\hline \multicolumn{5}{|l|}{ Healthcare utilization, number of visits ${ }^{b}$} \\
\hline \multicolumn{5}{|l|}{ Heart failure } \\
\hline IP & 82 & 75 & 65 & 61 \\
\hline OP & 112 & 101 & 88 & 82 \\
\hline ED & 18 & 17 & 14 & 13 \\
\hline$E D+I P$ & 4 & 4 & 4 & 3 \\
\hline \multicolumn{5}{|l|}{ Respiratory failure } \\
\hline IP & 5 & 4 & 4 & 3 \\
\hline OP & 2 & 1 & 1 & 1 \\
\hline ED & 0 & 0 & 0 & 0 \\
\hline$E D+I P$ & 0 & 0 & 0 & 0 \\
\hline \multicolumn{5}{|l|}{ Economic outcomes (US\$) } \\
\hline \multicolumn{5}{|l|}{ Cost of complications } \\
\hline \multicolumn{5}{|l|}{ Heart failure } \\
\hline IP & $\$ 1,013,747.81$ & $\$ 916,878.58$ & $\$ 802,550.35$ & $\$ 746,231.03$ \\
\hline OP & $\$ 5 I, 597.26$ & $\$ 46,666.86$ & $\$ 40,847.83$ & $\$ 37,981.32$ \\
\hline ED & $\$ 27,003.80$ & $\$ 24,423.44$ & $\$ 21,378.01$ & $\$ 19,877.80$ \\
\hline$E D+I P$ & $\$ 60,975.79$ & $\$ 55,149.21$ & $\$ 48,272.50$ & $\$ 44,884.96$ \\
\hline \multicolumn{5}{|l|}{ Respiratory failure } \\
\hline IP & $\$ 93,530.69$ & $\$ 83,000.83$ & $\$ 70,573.16$ & $\$ 64,451.15$ \\
\hline OP & $\$ 358.12$ & $\$ 317.80$ & $\$ 270.21$ & $\$ 246.77$ \\
\hline ED & $\$ 989.59$ & $\$ 878.18$ & $\$ 746.69$ & $\$ 681.92$ \\
\hline$E D+I P$ & $\$ 4,077.28$ & $\$ 3,618.25$ & $\$ 3,076.49$ & $\$ 2,809.61$ \\
\hline Total & $\$ 1,252,280.33$ & $\$ 1,130,933.14$ & $\$ 987,715.24$ & $\$ 917,164.55$ \\
\hline
\end{tabular}

(Continued) 
Table 3 (Continued).

\begin{tabular}{|c|l|l|l|l|}
\hline & \multicolumn{3}{|l|}{ PAC Adoption Rates } \\
\cline { 2 - 5 } & $\mathbf{0 . 0 \%}$ & $\mathbf{3 4 . 4 \%}$ & $\mathbf{7 5 . 0 \%}$ & $\mathbf{9 5 . 0 \%}$ \\
\hline $\begin{array}{l}\text { Overall economic outcomes summary (US\$) } \\
\text { Overall cost for annual cardiac surgical volume } \\
\text { Overall savings for annual cardiac surgical volume }\end{array}$ & & & \\
\end{tabular}

Notes: ${ }^{a}$ Annual cardiac surgical volume $=3,845$. ${ }^{b}$ /year and number of visits rounded to nearest unit. 'Savings to the integrated payer-provider system compared to $0 \%$ PAC adoption.

Abbreviations: IP, inpatient visit; OP, outpatient visit; ED, emergency department visit.

impact on savings were index treatment costs for RF and HF complications. As expected, higher treatment costs for complications were associated with larger cost savings. Decreased HF and RF complication rates during the year following index admission were responsible for a decrease in healthcare utilization. Although $\sim 80 \%$ of savings are accrued during the index visit, $\sim 20 \%$ of total savings are due to treatment of persistent complications during the one-year follow-up. Our study suggests that an upfront reduction in index admission complications may have important downstream economic consequences for an integrated health system.

Across studies reporting costs associated with PAC utilization in cardiac surgery patients, only two include direct cost comparisons between patients with or without a PAC. A retrospective analysis of cardiac surgical patients in the National Inpatient Sample (NIS) database by Chiang et $\mathrm{al}^{6}$ found that total charges (not hospital costs) were higher in the PAC group versus the no PAC group $(\$ 133,000$ and $\$ 125,000$, respectively) among patients that received bypass anastomosis for heart revascularization or valve repair or replacement procedures. The present study analyzed a broader range of procedure types. A retrospective cohort study by Ramsey et $\mathrm{al}^{5}$ among patients undergoing non-emergent $\mathrm{CABG}$ surgery identified from an administrative database of community hospitals found total hospital costs to be $\$ 1,402$ higher for patients who received a PAC compared to patients without a PAC, although this study was conducted over two decades ago. Although both studies controlled for confounding factors that could potentially have influenced the association of PAC use on the outcomes examined, none of these studies used a propensity score-matched cohort design. Additionally, these studies did not report a breakdown of the costs associated with cardiac surgery complications and did not characterize the cost during the year following cardiac surgery. Only Chiang et al ${ }^{6}$ analyzed a study population comparable to the one considered within the current study; however, the study did not report any details regarding the rates of cardiopulmonary complications and associated cost. The patient population analyzed in Ramsey et $\mathrm{al}^{5}$ is not directly comparable to the current study population as the authors only included non-emergent CABG surgery patients, whereas Shaw et $\mathrm{al}^{10}$ included both nonemergent and emergent cardiac surgeries in urban teaching/ non-teaching hospitals and academic centres.

The present economic models utilized inputs and assumptions which are representative of real-world US hospitals, ${ }^{10,23}$ and savings generated are, therefore, transferable to acute care hospitals and integrated payer-provider health systems with comparable numbers of annual cardiac procedures to the current study base cases. We calculated savings based on a sample of pre-defined scenarios; for hospitals and health systems performing more cardiac procedures and/or utilizing a monitoring PAC with a greater proportion of patients, greater savings would be expected. Further research is required to understand the economic benefits of PAC adoption in cardiac surgery patients from the patient and societal perspective (eg, quality of life, productivity) as well as cost savings that might be achieved beyond the first-year follow-up period.

\section{Limitations}

Several limitations affect the findings of this study. Our model only evaluated direct hospital costs and did not account for direct and indirect costs from a societal or patient perspective. Indirect costs associated with HF and RF complications (eg loss of productivity, transportation cost, costs for caregiver) and medication costs for managing these complications outside the hospital were not included in the models. Costs for medication administered during the index visit and during inpatient stays and emergency visits in the 1-year follow-up period are included in the total cost; however, medications prescribed in the outpatient setting would be expected to incur additional cost for the treatment of $\mathrm{HF}$ and RF complications. 
Our models do not include management techniques that may be utilized for the "no PAC" cohort, eg transesophageal echo or other techniques. Since only the cost of a PAC is included for the PAC cohort, the resulting estimated savings from the models are conservative.

Although most cost inputs were obtained from HCUP, a large national database, and therefore likely to accurately reflect the average cost across US hospitals, a minor limitation is that the exact savings/cost would depend on the cost at a specific hospital where care is provided.

Our model uses costs derived from HCUP for the cost of complications. A limitation of the HCUP database is that it does not provide cost granularity specific to the postoperative complication versus from other medical conditions. However, since all costs were sourced from HCUP and annual savings were calculated by subtracting the costs at a given $(>0 \%)$ PAC adoption rate from costs associated with $0 \%$ PAC adoption, any under- or overestimation should occur across both groups and should have minimal effect on estimated savings.

Another limitation is that these costs can change from year to year and therefore may affect savings. Variation in treatment costs for HF and RF, constituting the largest contributions to savings, would have a relatively large impact on total savings amounts compared to changes in other input parameters. This variation, however, was modelled within the presented sensitivity analyses.

The integrated payer-provider model assumes that the payer-provider owns the hospitals or facilities where care is provided and/or takes financial responsibility for the care provided. Systems that do not own the site of care will most likely have other costing methods in place and savings will differ for these types of systems. Due to the lack of RCT data to inform our economic evaluation, we also acknowledge the limitation that our findings are contingent upon future validation of the efficacy of PAC use in a cardiac surgical population through additional well-conducted studies and the confirmation of PAC clinical benefit in these studies.

\section{Conclusion}

Both acute care hospitals and integrated payer-provider health systems can achieve significant economic savings by utilizing a PAC with adult cardiac surgery patients to guide clinical treatment. The main driver of these cost savings is the reduction in cardiopulmonary complications which leads to lower overall treatment costs. Despite significant mid-term healthcare utilization associated with cardiopulmonary complications, the bulk of the treatment costs is incurred during the index surgical stay. Organizations with a high PAC adoption rate for their cardiac surgery population may observe increased cost savings.

\section{Acknowledgments}

The authors wish to thank Rita Tavares for assistance with manuscript preparation and Brian Nathanson for statistical assistance.

\section{Funding}

Funding for this research was provided by Edwards Lifesciences, Irvine, CA. The funders (other than the named authors) had no role in study design, data collection, decision to publish, or preparation of the manuscript. The funder had no role in data analysis.

\section{Disclosure}

Mitali Stevens and Todd Davis report being an employee of Edwards Lifesciences, during the conduct of the study and outside the submitted work. Sibyl H Munson reports working as a consultant for Boston Strategic Partners, Inc., who received fees from Edwards Lifesciences to support this research, during the conduct of the study. Apeksha $\mathrm{V}$ Shenoy, Boye LA Gricar, and Halit O Yapici report being employees of Boston Strategic Partners who received payment from Edwards Lifesciences to conduct this research, during the conduct of the study. Andrew D Shaw reports personal fees from Edwards Lifesciences, outside the submitted work, and works as a consultant for Edwards Lifesciences. The authors report no other potential conflicts of interest for this work.

\section{References}

1. D'Agostino RS, Jacobs JP, Badhwar V, et al. The society of thoracic surgeons adult cardiac surgery database: 2018 update on outcomes and quality. Ann Thorac Surg. 2018;105(1):15-23. doi:10.1016/j.athoracsur. 2017.10.035

2. Brovman EY, Gabriel RA, Dutton RP, Urman RD. Pulmonary artery catheter use during cardiac surgery in the United States, 2010 to 2014. $J$ Cardiothorac Vasc Anesth. 2016;30(3):579-584. doi:10.10 53/j.jvca.2015.11.012

3. Greenberg SB, Murphy GS, Vender JS. Current use of the pulmonary artery catheter. Curr Opin Crit Care. 2009;15(3):249-253. doi:10.1097/MCC.0b013e32832b302b

4. Judge O, Ji F, Fleming N, Liu H. Current use of the pulmonary artery catheter in cardiac surgery: a survey study. $J$ Cardiothorac Vasc Anesth. 2015;29(1):69-75. doi:10.1053/j.jvca.2014.07.016

5. Ramsey SD, Saint S, Sullivan SD, Dey L, Kelley K, Bowdle A. Clinical and economic effects of pulmonary artery catheterization in nonemergent coronary artery bypass graft surgery. $J$ Cardiothorac Vasc Anesth. 2000;14(2):113-118. doi:10.1016/S1053-0770(00)90001-6 
6. Chiang Y, Hosseinian L, Rhee A, Itagaki S, Cavallaro P, Chikwe J. Questionable benefit of the pulmonary artery catheter after cardiac surgery in high-risk patients. J Cardiothorac Vasc Anesth. 2015;29 (1):76-81. doi:10.1053/j.jvca.2014.07.017

7. Larson LO, Kyff JV. The cost-effectiveness of Oximetrix pulmonary artery catheters in the postoperative care of coronary artery bypass graft patients. J Cardiothorac Anesth. 1989;3(3):276-279. doi:10.10 16/0888-6296(89)90107-5

8. Pearson KS, Gomez MN, Moyers JR, Carter JG, Tinker JH. A cost/ benefit analysis of randomized invasive monitoring for patients undergoing cardiac surgery. Anesth Analg. 1989;69(3):336-341. doi:10.1213/00000539-198909000-00011

9. Xu F, Wang Q, Zhang H, Chen S, Ao H. Use of pulmonary artery catheter in coronary artery bypass graft. Costs and long-term outcomes. PLoS One. 2015;10(2):e0117610. doi:10.1371/journal.pone.0117610

10. Shaw AD, Mythen MG, Shook D, et al. Pulmonary artery catheter use in adult patients undergoing cardiac surgery: a retrospective, cohort study. Perioperative Med. 2018;7(1):24. doi:10.1186/s13741018-0103-x

11. Kilgore ML, Ghosh K, Beavers CM, Wong DY, Hymel PA Jr, Brossette SE. The costs of nosocomial infections. Med Care. 2008;46(1):101-104. doi:10.1097/MLR.0b013e3181468991

12. Riu M, Chiarello P, Terradas R, et al. Incremental cost of nosocomial bacteremia according to the focus of infection and antibiotic sensitivity of the causative microorganism in a university hospital. Medicine. 2017;96:17. doi:10.1097/MD.0000000000006 645

13. Nicholson G, Gandra SR, Halbert RJ, Richhariya A, Nordyke RJ. Patient-level costs of major cardiovascular conditions: a review of the international literature. Clin Econ Outcomes Res. 2016;8:495. doi:10.2147/CEOR.S89331

14. Mehaffey JH, Hawkins RB, Byler M, et al. Cost of individual complications following coronary artery bypass grafting. J Thorac Cardiovasc Surg. 2018;155(3):875-882. doi:10.1016/j.jtcvs.2017.08.144

15. Yoon J, Fonarow GC, Groeneveld PW, et al. Patient and facility variation in costs of VA heart failure patients. JACC Heart Fail. 2016;4(7):551-558. doi:10.1016/j.jchf.2016.01.003

16. Voigt J, John MS, Taylor A, Krucoff M, Reynolds MR, Michael Gibson C. A reevaluation of the costs of heart failure and its implications for allocation of health resources in the United States. Clin Cardiol. 2014;37(5):312-321. doi:10.1002/clc.22260
17. Gray A, Goodacre S, Newby D, et al. A multicentre randomised controlled trial of the use of continuous positive airway pressure and non-invasive positive pressure ventilation in the early treatment of patients presenting to the emergency department with severe acute cardiogenic pulmonary oedema: the 3CPO trial. Health Technol Assessment. 2009;13(33):1-106. doi:10.3310/hta13330

18. Vitacca M, Bianchi L, Guerra A, et al. Tele-assistance in chronic respiratory failure patients: a randomised clinical trial. Eur Respir J. 2009;33(2):411-418. doi:10.1183/09031936.00005608

19. Smith DA, Nehring SM. Bacteremia. StatPearls [Internet]: StatPearls Publishing; 2017.

20. Trautner BW. Management of catheter-associated urinary tract infection. Curr Opin Infect Dis. 2010;23(1):76-82. doi:10.1097/QCO.0b 013e328334dda8

21. Stevens M, Shenoy AV, Munson SH, et al. Healthcare utilization and costs of cardiopulmonary complications following cardiac surgery in the United States. PLoS One. 2019;14(12):e0226750. doi:10.1371/ journal.pone.0226750

22. Ediom. MarketPulse report.2018.

23. HCUP Databases, Healthcare Cost and Utilization Project (HCUP) [Internet]. 2018. Available from: www.hcup-us.ahrq.gov/databases. jsp. Accessed January 15, 2021.

24. CPI Inflation Calculator [Internet]. Availablefrom: https://www.bls. gov/data/inflation_calculator.htm. Accessed January 15, 2021.

25. Kaiser Permanente: who-we-are/fast-facts. Available from: https:// about.kaiserpermanente.org/who-we-are/fast-facts. Accessed April 5, 2019.

26. California Cardiac Surgery Intervention Project, Heart Surgery and Intervention Outcomes for California Hospitals [Internet]. 2018. Available from: https://www.californiacardiacsurgery.com/ CCSIP2018/. Accessed January 15, 2021.

27. Wilson L, Devine EB, So K. Direct medical costs of chronic obstructive pulmonary disease: chronic bronchitis and emphysema. Respir Med. 2000;94(3):204-13.

28. Physician Fee Schedule [internet] 2018. Available from: https://www.cms. gov/Medicare/Medicare-Fee-for-Service-Payment/PFSlookup. Accessed April 2019.

29. Hozo SP, Djulbegovic B, Hozo I. Estimating the mean and variance from the median, range, and the size of a sample. BMC Med Res Methodol. 2005;5:13. doi:10.1186/1471-2288-5-13
ClinicoEconomics and Outcomes Research

\section{Publish your work in this journal}

ClinicoEconomics and Outcomes Research is an international, peerreviewed open-access journal focusing on Health Technology Assessment, Pharmacoeconomics and Outcomes Research in the areas of diagnosis, medical devices, and clinical, surgical and pharmacological intervention. The economic impact of health policy and health systems organization also constitute important areas of coverage. The manuscript management system is completely online and includes a very quick and fair peer-review system, which is all easy to use. Visit http://www.dovepress.com/testimonials.php to read real quotes from published authors. 